SHS Web of Conferences 24, 01011 (2016)

DOI: $10.1051 /$ shsconf/ 20162401011

(C) Owned by the authors, published by EDP Sciences, 2016

\title{
Review of safety community construction strategy in China
}

\author{
Zhulin Li \\ Tianjin University, Tianjin, China
}

\begin{abstract}
The introduction and development of safety community is an inevitable trend of the economic and social development and an inevitable requirement of safety development in China. However, the construction of the safety community in China still has shortcomings and improvements. This paper describes the basic concept of the safety community, analyzes the development of the construction of domestic and foreign safety communities and research results of the construction of safety communities, and introduces the World Health Organization's recognized standards and relevant indicators of the safety community. What's more, this paper combined with the features of China's safety communities also analyzes problems and shortcomings about the construction of safety communities in China and puts forward recommendations and countermeasures for the construction of China's safety communities.
\end{abstract}

Keywords: safety community; influencing factors; strategy

\section{INTRODUCTION}

In recent years, people pay more attention to the safety issues. According to the survey, 700,000 people die from various kinds of injuries per year in China, accounting for about $11 \%$ of all deaths. In recent years, in China, there are more than 1 million types of accidents in the field of safety production, more than 130,000 deaths and more than 700,000 injured people. The expected direct economic losses caused by the accidents are 250 billion yuan. And the production accidents lead to huge losses to China's sustainable development capacity ${ }^{[1]}$.

The community is the critical point to solve social problems, which plays an immeasurable role in maintaining the social harmony and resolving social contradictions.

With the continuous development of the economic society and increase of the degree of social complexity, how to effectively prevent and reduce various kinds of hazards and improve the degree of social stability has become a hot issue to concern around the world.

The safety community is characterized by preventive, advanced, systematic and integrated control. The fact proves that, the construction work of the safety community plays a significant role in reducing accident disasters and improving personal safety. The community is the most basic level of organization, which can achieve the resources integration by strengthening this organization and boost the resources integration, continuous improvement, allpeople participation and other concepts of safety community. Therefore, it becomes an important driving force to strengthen the safety production and promote the social construction and innovation management. However, due to the national conditions of China, the relevant theoretical system of the safety community has been not yet mature, the risk control planning issues, cross-border organization construction issues and other key issues have been not yet completely resolved, and the quality of the safety community needs to be improved ${ }^{[2]}$.

The concept of safety community (SC) was first proposed by the World Health Organization (WHO) in the "World's Accidents and Prevention Conference" held in Stockholm, Sweden in 1989. The original intention of the safety community is to unite various strengths of the community to improve the community safety, and let the personal safety and health of the community residents be guaranteed in daily life in the community or even at work, entertainment places and other occasions. What's more, it also aims at lowering various safety hazards to a minimum level. And the objects of the safety community are very broad, covering everyone in the community.

For the safety community, the World Health Or- 
ganization proposes the relevant standards. That is, there is a need to achieve the following six basic requirements ${ }^{[3]}$ :

(1) There is a need to set up a trans-boundary organization to be responsible for the prevention of injuries, so as to comprehensively plan safety promotion matters in the community with a partnership mode.

(2) There is a need to continuously implement various promotion programs for a long term, and take into account different ages, genders, environment and situation

(3) There is a need to pay attention to high-risk people, high-risk environment and safety issues of the social vulnerable groups.

(4) There is a need to establish a mechanism to record the number and causes of injuries emerged.

(5) There is a need to establish appropriate indicators to assess the effectiveness and impact of the project.

(6) There is a need to actively participate in the exchange of experience of the local and international safety community networks.

In addition, the community safety promotion and cooperation center of the World Health Organization has developed 63 specific indicators based on the above six standards. And the construction of the safety community is the construction of the human security capacity. One of indicators about the construction of harmonious socialist society is to enhance the ability to resist injuries of the entire population and promote the sustainable development of society.

In recent years, China has made great achievements in understanding and accepting the concept of safety community, and positively responding to the call and publicizing the concept of safety community, and promoting the safety community work. China also vigorously learns and absorbs the experience of the construction of the foreign safety community, adheres to the scientific development concept and carries out the construction of the safety community.

\section{DOMESTIC AND FOREIGN RESEARCH STATUS OF SAFETY COMMUNITY}

The safety community plan is rapidly developed and widely recognized in developed and developing countries or regions in Europe and America after the first session of the World's Accidents and Prevention Conference. So far, thirteen sessions of the World Conference of Safety community have been held, and more than eighty safety communities have been officially named by WHO in the world.

Foreign scholars have investigated the advantages of safety community through a variety of ways. After 30 months of investigation on the safety community in Norway, Timpka B found that, the traffic accidents are obviously decreased with the gradual deepening of the construction of the safety community ${ }^{[4]}$, and the fall-down rate of the elderly is also declined; the construction of the safety community requires a certain cost, but the social benefits brought by the construction achievements preferably promote the economic growth ${ }^{[5]}$. Currently, foreign construction research on the safety community is mainly concentrated in three aspects, namely, the public security of community, culture and services ${ }^{[6]}$. For the community masses, the public security of community is the construction emphasis of the safety community. Therefore, the public security construction of community becomes one of the hotspot issues in the research of the construction of safety community ${ }^{[7-12]}$.

More safety communities are still in construction and gradually expanded to the developing countries, such as Indonesia, Bangladesh and other countries ${ }^{[13]}$ China's safety community construction and implementation norm is the Basic Requirements of Safety Community Construction drafted by the Occupational Safety and Health Association of China [14]. This standard refers to the international norm of safety community and combines with China's national conditions. The relations and differences between China's safety community and international safety community are shown in Table 1.

Suwon City in South Korea is the first Asian city with safety communities, which is named in the $11^{\text {th }}$ session of the World Safety Community Conference held in 2002

In China, Hong Kong Special Administrative Region takes the lead in carrying out the construction of safety communities. Hong Kong Occupational Safety and Health Council signed a contract with the community safety promotion and cooperation center of the World Health Organization on March 21, 2000, which has become the world's sixth safety community support center. The safety communities first confirmed by the World Health Organization are Tuen Mun community and Kwai Tsing community. Other communities have also been successively confirmed by the World Health Organization, such as Tai Po, Sham Shui Po and other communities. In Taiwan, four villages and towns, namely, Alishan Township in Chiayi County, Fengbin Township in Hualien County, Dongshi Township in Taichung County and Neihu District in Taipei City started to launch the safety community planning in 2002 and obtained confirmation by the World Health Organization in 2005.

In the Mainland of China, the National Security Supervision Bureau attaches great importance to the establishment and promotion of public safety culture. The first safety community in the Mainland of China sent an application to the community safety promotion and cooperation center of the World Health Organization in May 2005, and it was approved as a full member of the international safety community in March of the following year. As shown in Figure 1, with more and more attention to the safety community, 238 communities worldwide have been awarded as inter- 
Table 1. Contrast of characteristics of China's and international safety community

\begin{tabular}{|c|c|c|}
\hline Contrast item & International safety community & China's safety community \\
\hline Basic idea & \multicolumn{2}{|c|}{ Similarity: focus on carrying out safety work in a structured and programmed way; } \\
\hline $\begin{array}{l}\text { Characteristics } \\
\text { and ideas }\end{array}$ & $\begin{array}{l}\text { Resources integration } \\
\text { Community participation } \\
\text { Continuous improvement }\end{array}$ & $\begin{array}{l}\text { Fully integrate with China's national conditions } \\
\text { Absorb the concept of the international safety } \\
\text { community } \\
\text { Emphasize continuous improvement } \\
\text { Emphasize resources integration } \\
\text { Emphasize project leading } \\
\text { Emphasize all-people participation } \\
\text { Introduce PDCA model }\end{array}$ \\
\hline Core objective & \multicolumn{2}{|l|}{ Everyone is equal, and pursues safety and health } \\
\hline $\begin{array}{l}\text { Intervention } \\
\text { object }\end{array}$ & $\begin{array}{l}\text { Focus on solving the injury prevention with slighter } \\
\text { consequences and higher frequency }\end{array}$ & All-around intervention \\
\hline $\begin{array}{l}\text { Elements and } \\
\text { indicators }\end{array}$ & Seven standard requirements & 50 work indicators and 12 work elements \\
\hline Method & $\begin{array}{l}\text { Injury epidemiology; } \\
\text { Evidence-based management; } \\
\text { Injury prevention and control }\end{array}$ & $\begin{array}{l}\text { Systems engineering approach; } \\
\text { Evaluation of risk identification and control; } \\
\text { Management of safety objective; } \\
\text { Multi-central governance theory }\end{array}$ \\
\hline Safety field & $\begin{array}{l}\text { Nine items: school safety, elderly safety, traffic safety, } \\
\text { wading safety, sports safety, workplace safety and } \\
\text { home safety }\end{array}$ & $\begin{array}{l}\text { Twelve items: fire safety, disaster prevention and } \\
\text { mitigation and environmental safety, and social } \\
\text { security }\end{array}$ \\
\hline $\begin{array}{l}\text { Risk control } \\
\text { mode }\end{array}$ & $\begin{array}{l}\text { Combination with intervention and safety promotion } \\
\text { for all people with different sex and high-risk people, } \\
\text { high-risk environment and vulnerable group }\end{array}$ & $\begin{array}{l}\text { Combination with daily operation management, } \\
\text { project control and emergency plan and response }\end{array}$ \\
\hline $\begin{array}{l}\text { Organization } \\
\text { mode }\end{array}$ & $\begin{array}{l}\text { Partner cooperation, NGO and spontaneous organiza- } \\
\text { tion mode }\end{array}$ & Cross-border participation, government-led mode \\
\hline
\end{tabular}

national safety communities by August 2012, of which 74 communities in our country, 9 in Hong Kong, 19 in Taiwan and 46 in the Mainland of China ${ }^{[15-17]}$.

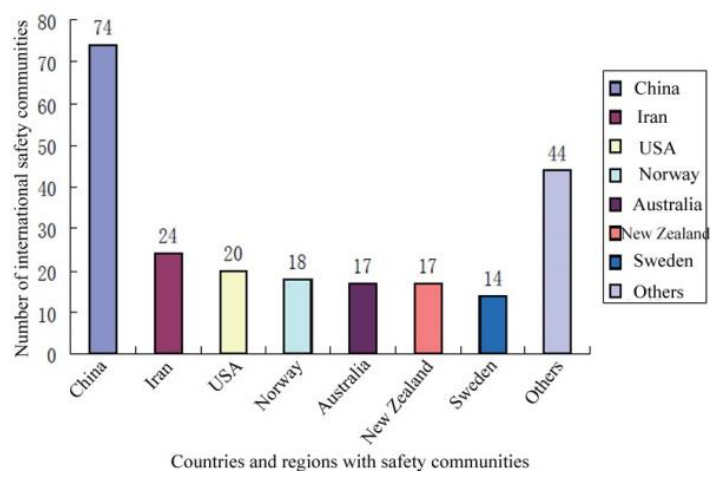

Figure 1. Distribution diagram of the countries and regions with international safety communities

\section{PROBLEMS AND SHORTCOMINGS OF CHINA'S SAFETY COMMUNITY}

From the perspective of our country's construction of safety communities, it still has many problems even if it has already achieved a good performance.

3.1 Construction standards of safety community need to be improved to adapt to China's actual conditions

To construct safety communities, there is a need to establish a reasonable working mechanism. According to China's national conditions, China's safety criterion with respect to the construction standards of the international safety community require to pay more attention to the management content and to be more systematic. However, currently, the work in this regard should be further strengthened. It requires to gradually explore and summarize the construction and development mode that is in line with China's economic and social level at the present stage, and also requires to strengthen leadership force, joint construction and common participation of the party and government departments. In practice, there are still many problems in the establishment of such reasonable working mechanism, which shall be further strengthened.

\subsection{Community organizations need to further play an autonomous role}

With the gradual fading of the duties and functions of the "unit", the significance and influence of the community gradually increase. The construction of the safety community is an important part of the community work, and the security and stability of the community directly affects the entire society. The construction of the safety community is an essential factor to promote the economic development, build a harmonious society and maintain social stability in the transformation period of society. Currently, the community is lack of involvement in the autonomous organizations. Therefore, the construction of the safety community should strengthen the organizational management, education guidance, funding, exchange 
and cooperation. To truly give play to the advantages of the community and develop self-control ability of the community is a very important direction and content of the construction of the safety community ${ }^{[18-19]}$.

\subsection{The grass-root government regulation needs to be further strengthened}

The investigation and statistics show that, the accident rate of the grassroots is higher than that of the cities, and more than $60 \%$ of accidents happen in the towns and streets and other grassroots. From a legal perspective, the towns and sub-district offices need to receive the supervision and management of superior people's government, and do not have relevant jurisdiction for supervision and management of the safety production. The effect of the construction of the grassroots safety community determines the effect of China's overal situation of safety production. Therefore, combined with the work of other related social organizations, the relevant safety supervision department should take effective measures to strengthen the safety supervision of the communities.

\section{STRATEGY OF CHINA'S SAFETY COMMUNITY CONSTRUCTION}

For the problems and shortcomings of China's safety community, it carries out a lot of research work for the safety community based on the absorption of the experience in the construction of foreign safety communities $^{[20-27]}$

\subsection{Combine the construction of the safety community with people-oriented concept}

The starting point and standpoint of the safety community is people, so its construction is rooted in people. In the process of the construction of safety community, there is a need to always put the people's needs in the first place, think what the masses think and concern what the masses concern, and pay close attention to people's judgment and put guarantee for the safety of masses in the community in the first place $^{[20-22]}$

First, the people-oriented concept is embodied in the direction of the construction of safety community. It is specifically embodied as follows: select the most concerned and the most interesting issues for the masses as the construction target and project of the safety community; take a variety of ways to collect opinions and suggestions of the community residents, concern about the needs of the masses and ensure that the construction of the safety community always aims at the improvement of the quality of life of the people.

Second, the people-oriented concept is embodied in the process of construction of safety community. In the process of construction of safety community, there is a need to fully consider the feelings of the masses, strive for the support of the masses, communicate and interact with the masses, and mobilize the masses to participate in the process of the construction of safety community. The people-oriented concept and idea are embodied in the beneficial scope of the safety community ${ }^{[23]}$

\subsection{Strengthen the community governance level}

The community management level is a key to the construction of the safety community. Only to strengthen the community management level and unite the overall strength of the community can the safety community be constructed better.

The first thing we need to strengthen the community management level is to improve the community environment. There is a need to make rational planning and strengthen the community infrastructure, particularly the safety construction. The concept of safety community is embodied in the process of construction of community infrastructure. The safety warning labels shall be set up in critical areas, so as to strengthen software and hardware facilities of the community public security, and prevent all kinds of disasters and unsafe events ${ }^{[24,25]}$. Second, there is a need to do community accident statistics, set up a special organization to do real-time monitoring and periodic statistics of the community safety and injury accidents, and find the security flaws of the community through analysis and research of the data of the community safety incidents, and develop appropriate rectification plans for improvement. Meanwhile, there is also a need to test the construction achievement of the safety community through periodic statistics, so as to provide a good basis for development of better measures and further strengthen the construction of the safety community ${ }^{[26]}$

\subsection{Play a leading role of the government and form a join force with a variety of resources}

Currently, China is still subject to the government safety supervision department, and also responsible for promoting the construction and management of the safety community with the main objective of controlling over the safety accidents ${ }^{[27]}$. The government authority is still bound by single polarization, which greatly restricts the construction and development of safety community. The government needs to further emancipate the mind, streamline administration and delegate power to the lower levels, and strengthen cooperation with the society and market based on the actual situation of the community and combined with China's national conditions, so as to develop towards diversification and refinement route and target. At present, the allocation of social resources gradually converts from the previous simplification to diversification. To cope with such a situation, the safety com- 
munity is an important component of the social management and also a new carrier for social harmony and stability.

\subsection{Further improve the residents' safety culture quality}

The construction of safety community embodies the human's pursuit for the safety and health. This concept arouses a widespread recognition. Confronting the social risks, the first need is how to avoid physical and mental injury in the daily work and life.

The core point concerned in the construction of safety community is the vital interests, safety and health issues of the community residents. To analyze from the perspective of the safety culture, the key is harmonious development of the community. The community should vigorously promote the residents' safety education, adopt various forms of educational modes based on the safety needs of the residents to further enhance the safety awareness of the residents and improve the residents' safety quality. These are fundamental ways and long-term approaches to improve the community safety and enhance the construction effect of safety community.

\subsection{Further improve the involvement of the masses}

The construction of safety community is directly related to the community residents. The ultimate beneficiaries of the safety community are the masses in the community, so the masses should be the main participants in the construction of safety community. However, it is not a long-term plan to rely on the administration of the government to solve problems and propose requirements. The common people should actively participate in the development and construction of the safety community with the following three main channels: First, actively participate in the training related to the safety community and other cultural and sports activities organized by the community, and continuously improve the safety awareness and protection skills; second, offer advice and suggestions on the construction of safety community, and report the reasonable opinions and suggestions back to the appropriate organizations and departments through the relevant reasonable channels; third, join community organizations and provide all of best services to other people who need help in this area.

\section{CONCLUSION}

The community is a grass-root unit of the country, and the safe condition of the community is related to the vital interests of the masses. If the community's safety fails to be guaranteed, it is impossible to have a harmonious society. With the introduction and development of the safety community in China, people can obtain the good result of its achievement. However, if the concept of safety community is implemented well in China, it is a subject worthy of research due to the particularity of China's national situation. And this paper exactly introduces the domestic and foreign research status of the safety community, and researches the construction strategy of China's safety community by summarizing the problems and shortcomings of the construction of China's safety community.

\section{REFERENCES}

[1] Zhou Xiaozhong, Li Jinghua \& Zheng Suiling. 2010. Analysis of indigenous approach of the social work value from the perspective of value stratification. Journal of Northwestern Polytechnical University (Social Sciences), 30(1): 32-36

[2] Joon Pil cho \& Leif Svanstrom. 2002. Developing Safety community- Two decades of experiences. Department of Emergency Medicine Ajou University school of Medicine, Suwon, Korea, pp: 1-4.

[3] Wu Zongzhi. 2004. Introduction of WHO safety community construction standards. Journal of China Safety Production, 7(8): 8 .

[4] Ou Yangmei, Chen Wentao \& Duan Miao. 2006. Probe into methods of injury investigation and risk identification in establishing safety community. Journal of China Safety Science, 16(11): 92-97.

[5] Timpka B. 1999. Community based injury prevention: Effect on health care. Inter. J. Epidemiol., (28): 502.

[6] Lindqvist K. 2001. A cost-benefit analysis of the community-based injury prevention program in Montala, Sweden-a WHO safety community. Public Health, (115): 317.

[7] Peter Stoett. 1995. Human and Global Security: An Exploration of Tenns. Toronto: University of Toronto Press. pp: 148.

[8] Charles Guzzetta. 2001. The theory of human security and the task of training. Regional Development Dialogue, (22): 123.

[9] Mo Shuitu. 2014. Guangxi: A new pattern of public security prevention and control built in "one village with one police". People's Public Security News, 03-12 (5).

[10] Gao Yan. 2013. Reform and Development Outline of the Public Security Administration. Beijing: Chinese People's Public Security University Press, pp: 11.

[11] Wang Shiqing. 2010. Case Analysis of Basic Theory of Policing Science. Beijing: Chinese People's Public Security University Press, pp: 130-133.

[12] Li Chengwei. 2011. Construction of community safety governance mechanism--A case study of Neihu community safety promotion organization. Journal of Beijing University of Aeronautics and Astronautics (Social Sciences), 24(3): 18-23.

[13] Glenn Welander, Leif Svanstrom \& robert Ekman. 2004 Safety Promotion--An Introduction Karolinska Institute Department of Public Health Sciences Division of Social Medicine Stockholm.

14] Chen Qiang. 2004. Sustainable development of occupational health and safety management system-active upgrading of corporate safety culture. International JournalonArehiteeturalSeienee. 10 


\section{SHS Web of Conferences}

[15] Pierre Maurice, Julie Laforest \& Louise Marie Bouchard. 2008. Safety promotion and the setting-oriented approach. Theoretical and practical considerations. Quebec Safety Promotion and Crime Prevention Resource Centre

[16] Per Nilsen. 2007. The how and why of community-based injury prevention: A conceptual and evaluation model. Safety Science, 45(4): 501-521.

[17] Kent Lindqvist \& Per Nilsen. 2004. A manual for economic evaluation in safety community practice. Linköping: Linköping University, Department of Health and Society.

[18] Anna Halonen. 2005. Injury prevention and safety promotion-based on safety community principles in the Baltic sea region states. Aleksanteri Institute.

[19] Dong Chuanshi. 2011. Promotion of residents' safety awareness by safety intervention Youth Park Street in Huaiyin District of Jinan City_WHO safety community_promoting work. Modern Occupational Safety, 117: 94-97.

[20] Jin Lei. 2010. Safety community construction under the background of public safety. Modern Occupational Safety, 107: 106-110.
[21] Yu Jianhui, Zhang Wenzhong, Wang Dai \& Shen Li. 2011. Research progress of living environment safety based on the residents' perspective. Progress in Geography, 6: 699-705

[22] Liu Xiaojian \& Li Liping. 2010. Methods and application for quality assessment of injury surveillance system. Disease Surveillance, 25(3): 235-238.

[23] Yu Tao. 2012. Research on guidance and application of PDCA circulation theory in safety production management. Transportation World, 7: 306-307.

[24] Tan Jie. 2011. Discussion of how to create a safety community in grass-roots units. Modern Construction, 10(4): 38-40.

[25] Yang Yang \& Ye Yun. 2010. On the construction of urban safety community. Managers, 15: 184

[26] Gu Linsheng. 2011. Enlightenment of foreign grassroots emergency management. Chinese Emergency Management, 6: 36-40.

[27] Kuo Shoufong. 2011. Application of PDCA circulation in safety supervision and management. Construction Safety, 10: 21-22. 